\title{
Uncertainty analysis of dynamic thermal rating based on environmental parameter estimation
}

\author{
Yanling Wang ${ }^{1}$, Weihua Tao ${ }^{2 *}$, Zhijie Yan ${ }^{1}$ and Ran Wei $^{3}$
}

\begin{abstract}
Dynamic thermal rating (DTR) of transmission lines is related to wind speed, wind direction, ambient temperature, and so on. Among the environmental parameters, there is a difference between the obtained environmental parameters and the true value. Therefore, only the deterministic values of environmental parameters and DTR are not accurate enough. Considering the environmental parameters obtained with uncertainty, the uncertainty of environment parameters based on Monte Carlo Method (MCM) is studied in this paper. According to the heat balance equation of transmission lines, the uncertainty analysis of transmission line ampacity is realized based on CIGRE standard. The best estimation value, standard uncertainty, and confidence interval are obtained under a given confidence level of environmental parameters. The experimental results show that DTR can fully improve the transmission capacity of transmission lines, and MCM is an effective method to assess uncertainty of DTR.
\end{abstract}

Keywords: Transmission line, Dynamic thermal rating (DTR), Environmental parameters, Monte Carlo method (MCM), Uncertainty analysis

\section{Introduction}

Dynamic thermal rating (DTR) of transmission lines based on actual environmental parameters can greatly improve line capacity [1]. Without reconstructing the existing transmission lines, DTR can ease the contradiction between electricity consumption and power supply and improve line utilization with great economic benefits. DTR can be determined by line ampacity calculation model based on CIGRE standard [2-4]. The ambient environmental parameters of transmission lines are significant factors that affect the DTR, but the difference between the measured value and the true value cannot be ignored, and the uncertainty of DTR needs to be evaluated [5-8].

Guide to the expression of uncertainty in measurement (GUM) gives the basic method of assessing uncertainty $[9,10]$. However, the method is limited by certain conditions: (1) the probability distribution of the input quantity is assumed to be symmetrical, approximately normal distribution or $\mathrm{T}$ distribution; (2)

* Correspondence: twh77@sina.com

${ }^{2}$ Beaulieu Yarns (Weihai) Company Limited, Weihai, China

Full list of author information is available at the end of the article the probability distribution of the output is approximately normal or $\mathrm{T}$ distribution; (3) the measurement model is linear model or nonlinear model that can be reduced to linear model [7]. In 2008, the Joint Committee on Measurement Guidelines introduced a supplemental document. The Monte Carlo method (MCM) was used to assess measurement uncertainty [11-13]. According to the supplementary document, measurement uncertainty with the MCM is newly issued in China, which provides a method for assessing the uncertainty of measurement, thus broadening the application scope of uncertainty assessment.

In [14], the MATLAB method for evaluating random numbers in MCM was studied. The simulation of the relevant random variables was realized. It was concluded that MCM could overcome the shortcomings of GUM method in which it was difficult to evaluate the uncertainty of complex model. In [15, 16], the MCM evaluation uncertainty process was given, and the evaluation results of the GUM method were verified by MCM. The reliability of MCM uncertainty evaluation was proved. MCM can be applied to the situation where the GUM method is not 
applicable. To sum up, MCM is an effective method for uncertainty assessment. In this paper, MCM is used to evaluate the uncertainty of DTR of transmission lines.

This paper is organized as follows: Section 2 presents an extensive review of the uncertainty analysis of dynamic thermal rating. DTR of transmission lines based on CIGRE standard is introduced in detail in Section 3. In Section 4, we review the MCM and study the uncertainty of environmental parameters. In Section 5, after obtaining the uncertainty of the environmental parameters, we assess the uncertainty of DTR to ensure the reliability of the results. We conclude in Section 6.

\section{Methods}

The dynamic thermal rating is determined according to the real meteorological conditions of overhead lines according to wind speed, wind direction, and ambient temperature. The randomness of meteorological parameters and the existence of measurement errors all lead to uncertainty in the results of dynamic thermal rating. Therefore, it is not enough to give only a definite value of the current carrying capacity. It is necessary to give the uncertainty of the carrying capacity, and the result is more reliable. The dynamic thermal rating method based on CIGRE standard is studied. The Monte Carlo method is proposed to analyze and calculate the carrying capacity of the overhead transmission line.

\section{DTR method based on CIGRE standard}

This section briefly describes the CIGRE method of calculating DTR of overhead transmission lines. The steady state thermal balance equation of CIGRE standard is:

$$
I^{2} R_{\mathrm{ac}}\left(T_{\mathrm{c}}\right)+Q_{\mathrm{s}}=Q_{\mathrm{c}}+Q_{\mathrm{r}}
$$

where the convection heat is $Q_{c}$, radiation heat is $Q_{p}$ sunshine heat absorption is $Q_{\mathrm{s}}$, and the Joule heat is $I^{2} R_{\mathrm{ac}}$ generated by its own current, and $T_{\mathrm{c}}$ is the line conductor temperature. According to direct current (DC) resistance at $20{ }^{\circ} \mathrm{C}$, to find the alternate current (AC) resistance at $T_{\mathrm{c}}$ is $R_{\mathrm{ac}}\left(T_{\mathrm{c}}\right)=k_{\mathrm{j}} R_{\mathrm{dc}}\left[1+\alpha\left(T_{\mathrm{c}}-20\right)\right], k_{\mathrm{j}}$ usually takes as $1.0123, R_{\mathrm{dc}}$ is the DC resistance of the line, and $\alpha$ is the resistance temperature coefficient. The convection heat dissipation is shown in Eq. (2).

$$
Q_{\mathrm{c}}=\pi k_{\mathrm{cf}}\left(T_{\mathrm{c}}-T_{\mathrm{a}}\right) K_{\mathrm{angle}} N_{\mathrm{u}}
$$

where $k_{\mathrm{cf}}=2.42 \times 10^{-2}+3.6 \times 10^{-5} \times\left(T_{\mathrm{c}}+T_{\mathrm{a}}\right)$ is the ambient air thermal conductivity, $T_{\mathrm{a}}$ is the ambient temperature, and $K_{\text {angle }}$ is coefficient of wind direction. Convection heat dissipation is also divided into two
Table 1 Nusselt number parameters

\begin{tabular}{llll}
\hline Surface roughness & Reynolds range & $B_{1}$ & $n$ \\
\hline Various surface & $\left(10^{2}, 2.65 \times 10^{3}\right)$ & 0.641 & 0.471 \\
$R_{\mathrm{f}} \leq 0.05$ & $\left(2.65 \times 10^{3}, 5 \times 10^{4}\right)$ & 0.178 & 0.633 \\
$R_{\mathrm{f}}>0.05$ & $\left(2.65 \times 10^{3}, 5 \times 10^{4}\right)$ & 0.048 & 0.800 \\
\hline
\end{tabular}

cases of high wind speed and low wind speed, where the Nusselt number is $N_{\mathrm{u}}$ and $N_{\mathrm{u}}=B_{1}\left(R_{\mathrm{e}}\right)^{n}$. $R_{\mathrm{e}}$ is the Reynolds number as shown in Eq. (3).

$$
R_{\mathrm{e}}=\frac{D \rho_{0} \exp \left(-1.16 \times 10^{-4} H_{\mathrm{e}}\right) V_{\mathrm{w}}}{1.32 \times 10^{-5}+4.75 \times 10^{-8}\left(T_{\mathrm{c}}-T_{\mathrm{a}}\right)}
$$

where $D$ is the line diameter, $\rho_{0}$ is air density at the sea level, $V_{\mathrm{w}}$ is wind speed, $H_{\mathrm{e}}$ is the line altitude, $B_{1}$ and $n$ is decided by $R_{\mathrm{e}}$ and the line surface roughness $R_{\mathrm{f}}=d /$ $[2(D-2 d)](d$ is the outer diameter) as shown in Table 1 . $D$ is $27.63 \mathrm{~mm}$ and $d$ is $3.07 \mathrm{~mm}$ for the transmission line of LGJ-400/50.

The CIGRE standard also takes into account the effects of wind direction on $Q_{c}$, the correction factor is $K_{\text {angle }}=A_{1}+B_{2} \sin (\phi)^{m 1}$. When the angle between the wind and the line is $0^{\circ} \leq \phi \leq 24^{\circ}$, then $A_{1}=0.42, B_{2}=0.68$, $m_{1}=1.08$. When the angle is $24^{\circ} \leq \phi \leq 90^{\circ}$, then $A_{1}=$ $0.42, B_{2}=0.58$, and $m_{1}=0.9$. When there is no wind, the number of Nusselt is determined by the value of $G_{\mathrm{r}}$ and the value of $P_{\mathrm{r}}, N_{\mathrm{u}}=A_{2}\left(G_{\mathrm{r}} \times P_{\mathrm{r}}\right)^{m_{2}} . P_{\mathrm{r}}$ and $G_{\mathrm{r}}$ are shown in Eqs. (4) and (5).

$$
\begin{aligned}
& P_{\mathrm{r}}=0.715-1.25 \times 10^{-4}\left(T_{\mathrm{c}}+T_{\mathrm{a}}\right) \\
& G_{\mathrm{r}}=\frac{D^{3} \rho_{o}^{2}\left(T_{\mathrm{c}}-T_{\mathrm{a}}\right) g}{\left[\left(T_{\mathrm{c}}+T_{\mathrm{a}}\right) / 2+273\right] \mu_{\mathrm{f}}^{2}}
\end{aligned}
$$

where $g=9.8 \mathrm{~m} / \mathrm{s}^{2}$ and $A_{2}, m_{2}$ are determined by $G_{\mathrm{r}} \times P_{\mathrm{p}}$, which are shown in Table 2.

The radiation heat dissipation is shown in Eq. (6).

$$
Q_{\mathrm{r}}=0.0178 D \varepsilon\left[\left(\frac{T_{\mathrm{c}}+273}{100}\right)^{4}-\left(\frac{T_{\mathrm{a}}+273}{100}\right)^{4}\right]
$$

where $\varepsilon$ represents the radiation coefficient of transmission line, ranging from 0.23 to 0.91 ; $\varepsilon$ is 0.23 for the new transmission lines; and $\varepsilon$ is 0.91 for the long life lines. Radiation heat is decided by the line

Table 2 The value of parameters $A_{2}$ and $m_{2}$

\begin{tabular}{lll}
\hline$G_{r} \times P_{r}$ & $A_{2}$ & $m_{2}$ \\
\hline$\left(10^{-1}, 10^{2}\right]$ & 1.020 & 0.148 \\
$\left(10^{2}, 10^{4}\right]$ & 0.850 & 0.188 \\
$\left(10^{4}, 10^{7}\right]$ & 0.480 & 0.250 \\
$\left(10^{7}, 10^{12}\right]$ & 0.125 & 0.333 \\
\hline
\end{tabular}


diameter, conductor temperature, ambient temperature, and radiation cooling coefficient. The greater the radiation heat is, the more help to improve the transmission capacity of the line.

The heat absorption in the CIGRE standard takes into account the absorption of direct sunlight, the absorption of albedo sunshine and the absorption of solar heat dissipation, as shown in Eq. (7).

$$
Q_{\mathrm{s}}=\alpha_{\mathrm{s}} D\left[I_{\mathrm{D}}\left(\sin \theta+\frac{\pi}{2} F \sin H_{\mathrm{c}}\right)+(\pi / 2) I_{\mathrm{d}}(1+F)\right]
$$

where $I_{\mathrm{D}}=1280 \sin H_{\mathrm{s}} /\left(\sin H_{\mathrm{s}}+0.314\right)$ is the absorption of direct sunlight heat. $F$ is albedo growing with $H_{\mathrm{c}} \cdot I_{\mathrm{d}}$ is sun heat dissipation. In sunny weather conditions, it is the $10 \%$ of $I_{\mathrm{D}}$. The DTR under actual environmental

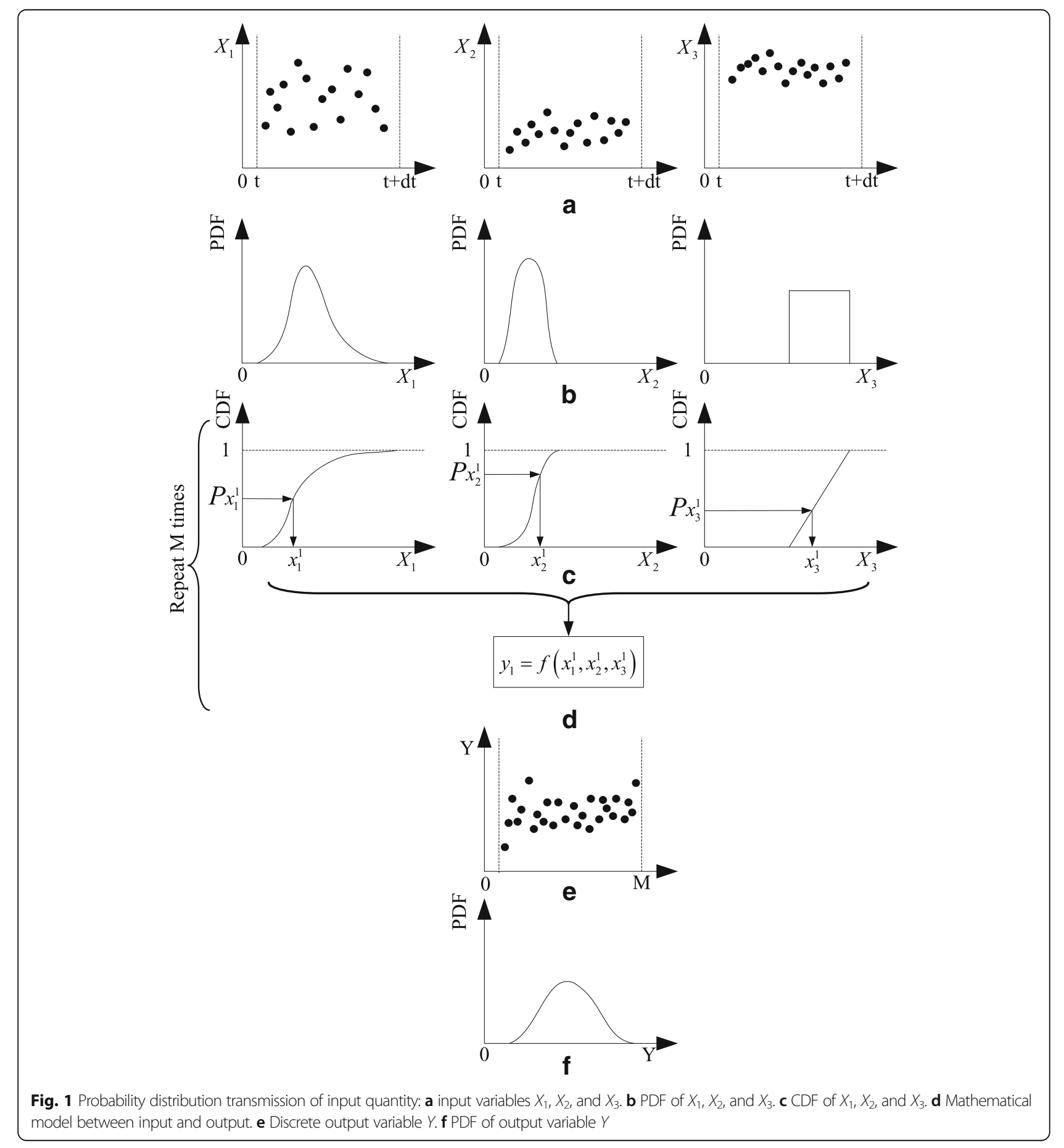




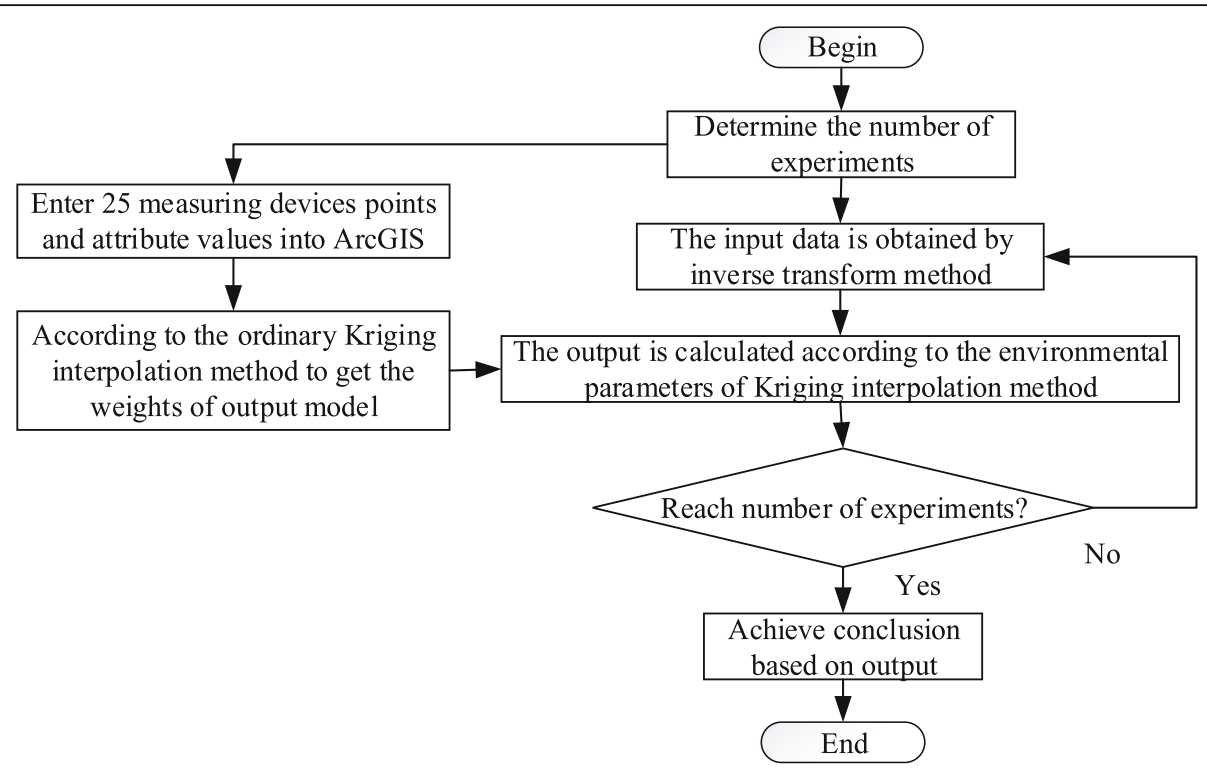

Fig. 2 Calculation procedure for uncertainty of environmental parameters

parameters is taken into account when the steady state equilibrium is deduced from Eq. (1), and the ampacity is calculated in Eq. (8).

$$
I=\sqrt{\frac{Q_{\mathrm{s}}-Q_{\mathrm{c}}-Q_{\mathrm{r}}}{R_{\mathrm{ac}}\left(T_{\mathrm{c}}\right)}}
$$

\section{Monte Carlo method}

The MCM is known as a random simulation method or a statistical testing method. It is based on the stochastic sampling. By means of random sampling, the random number in the corresponding distribution of the random variables is repeatedly selected. The stochastic number satisfying the particular distribution is obtained as the input data. The discrete value of the output is calculated by solving model. Then, the best estimated value, the standard uncertainty, and the corresponding inclusion interval under a given confidence level are acquired from the statistical results of the output value. MCM is an effective

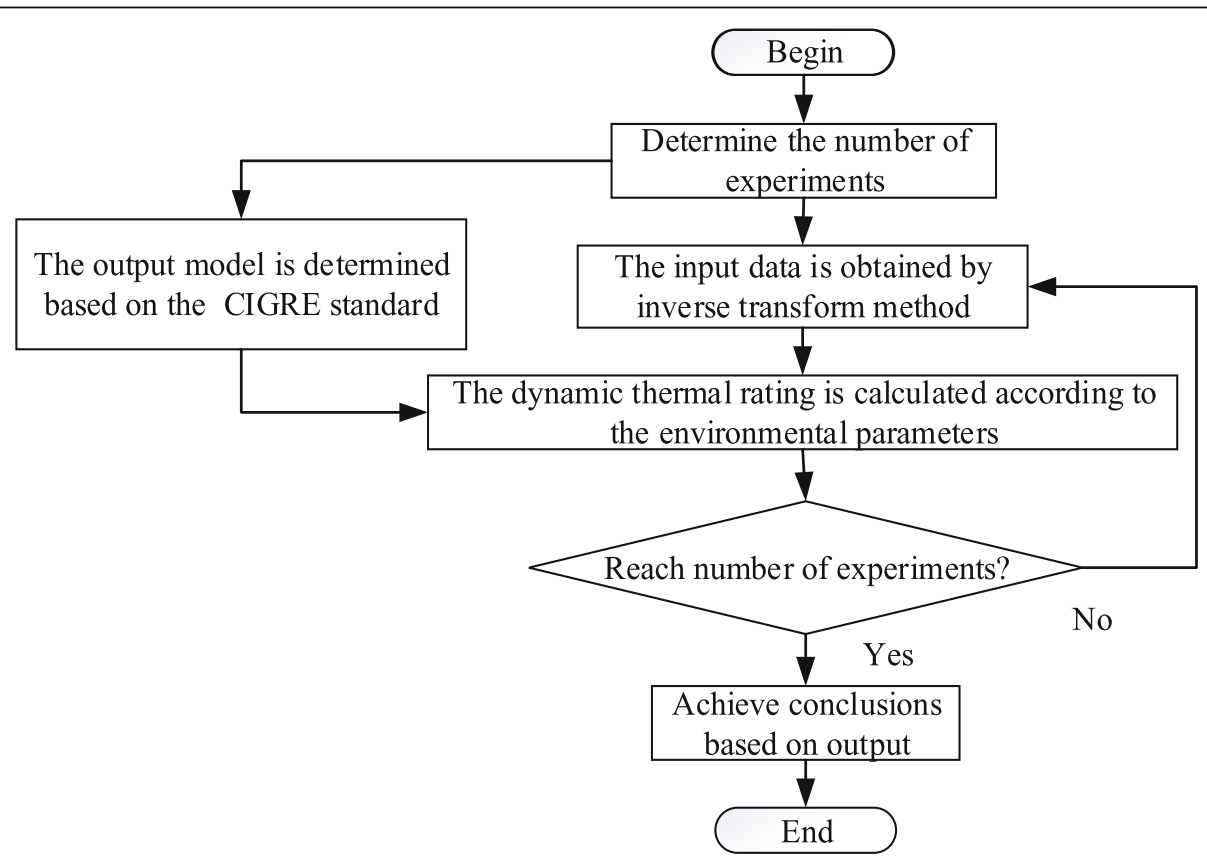

Fig. 3 Calculation procedure for uncertainty of DTR 
solution for some complex models which are difficult to calculate for an analytic solution.

\subsection{Process of MCM to solve the uncertainty problems}

Solving uncertainty problems with MCM usually involves three steps: The first step is model building. By analyzing the problem, the mathematical model between the output and the input is determined, and the number of experiments to be carried out by MCM is given.

The second step is probability distribution and transfer. By the probability density function of the input quantity, the random number is obtained from the inverse transformation method. The output quantity is obtained by substituting the random number as the input quantity into the mathematical model. Repeat this step and stop when the experiment number is reached.

The third step is statistical calculation. The best estimate value, the standard uncertainty, and the corresponding inclusion interval at the given confidence level are presented from statistical analysis of all the discrete outputs obtained by the model.
Suppose that the confidence interval corresponding to the output confidence level of $100 p \%$ is finally required. The number of MCM repeated calculations is $M$ times, and $M$ satisfies Eq. (9).

$$
M \geq \frac{1}{10 p} 10^{4}
$$

The distribution characteristics of the input quantity are transmitted through the corresponding transfer model, and the distribution characteristic of the output quantity can be obtained. It is assumed that the three inputs are independent. Figure 1a represents the value $\left(X_{1}, X_{2}, X_{3}\right)$ of the corresponding input in time $\mathrm{d} t$. Figure $1 \mathrm{~b}$ is the probability density function (PDF) corresponding to the three input quantities. Figure $1 \mathrm{c}$ is the cumulative density function (CDF) calculated from the PDF integral. The random input variables are obtained by $M$ times inverse calculation. Figure $1 \mathrm{~d}$ shows that the input variables obtained by the inverse calculation are substituted into the mathematical model to calculate

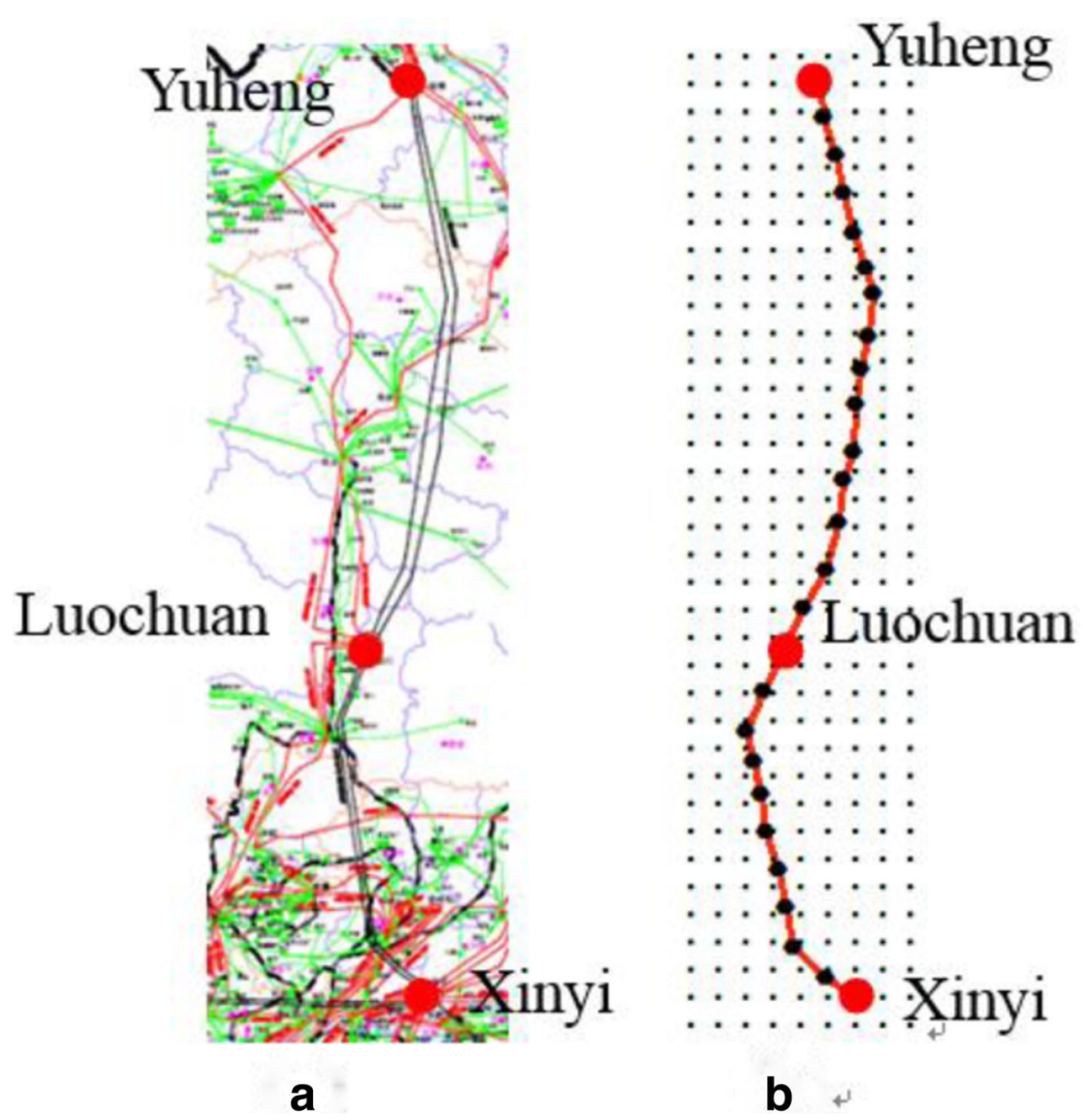

Fig. 4 The geographical location of the studied line: a geographic wiring diagram and $\mathbf{b}$ meteorological data network 
output variables. Figure 1e shows discrete output variables, and Fig. If is the corresponding PDF according to the discrete output variables. The optimal estimate value, the standard uncertainty and the inclusion interval under the given confidence level are obtained.

From Fig. 1, we can see that $X_{1}$ obeys the lognormal distribution, $X_{2}$ obeys the normal (Gaussian) distribution, and $X_{3}$ obeys the uniform distribution. The distribution function is sampled for $M$ times, and the sampled data as input variables is taken into the mathematical model to obtain discrete output variables, then the mean of output variables, the standard deviation, and the confidence level can be got.

\subsection{MCM for analyzing environmental parameters and DTR uncertainty}

The flow chart for solving the uncertainty of environmental parameters and DTR is shown in Figs. 2 and 3, respectively.

The location and attribute values of the points with known environment parameter are put into the geographic statistical analysis model in ArcGIS software. The estimating environmental parameter value of a point is obtained by Kriging interpolation. Experiments are repeated by MCM. After reaching the number of experiments, we count the discrete output of each experiment to get the best estimate, the standard uncertainty, and the corresponding interval endpoint with the confidence level of $100 p \%$.

In Fig. 3, the input data include wind speed, wind direction, and ambient temperature. The above input parameters are brought into the CIGRE standard heat balance equation, and the dynamic thermal rating of overhead lines can be obtained. As can be seen from Figs. 2 and 3, obtaining input data based on the inverse transform method is an important step in the MCM. If the distribution function $F(x)$ of random variable $X$ is continuous and $r=F(x)$ is set, then $r$ is a uniform random variable on the interval $(0,1)$. Therefore, the sampled value $x=F^{-1}(r)$ of the random variable $X$ obeys the corresponding distribution function. $F(x)$ can be obtained by extracting the random

Table 3 Longitude, latitude and environmental parameters of 25 known points

\begin{tabular}{|c|c|c|c|c|c|}
\hline Points & Longitude (E) & Latitude $(\mathrm{N})$ & Temperature $\left({ }^{\circ} \mathrm{C}\right)$ & Wind speed $(\mathrm{m} / \mathrm{s})$ & Wind direction $\left(^{\circ}\right)$ \\
\hline 1 & 109.676 & 37.989 & 22.31258 & 1.71582 & 156.3338 \\
\hline 2 & 109.686 & 37.884 & 22.67912 & 2.14189 & 149.2344 \\
\hline 3 & 109.729 & 37.744 & 23.01952 & 2.33556 & 143.2542 \\
\hline 4 & 109.758 & 37.609 & 23.21038 & 2.26855 & 138.0481 \\
\hline 5 & 109.792 & 37.470 & 23.42952 & 2.04048 & 131.4788 \\
\hline 6 & 109.835 & 37.340 & 23.60655 & 1.84029 & 127.6865 \\
\hline 7 & 109.864 & 37.244 & 23.64497 & 1.68302 & 125.5642 \\
\hline 8 & 109.844 & 37.095 & 23.60006 & 1.58664 & 125.6270 \\
\hline 9 & 109.820 & 36.970 & 23.35387 & 1.59052 & 124.9162 \\
\hline 10 & 109.801 & 36.845 & 22.94237 & 1.58255 & 126.9266 \\
\hline 11 & 109.792 & 36.671 & 22.68975 & 1.67703 & 136.2848 \\
\hline 12 & 109.758 & 36.570 & 22.96728 & 1.94498 & 143.1490 \\
\hline 13 & 109.739 & 36.416 & 23.63994 & 2.66577 & 155.6990 \\
\hline 14 & 109.695 & 36.243 & 23.97541 & 3.58796 & 166.6059 \\
\hline 15 & 109.614 & 36.113 & 23.72943 & 3.75889 & 174.8989 \\
\hline 16 & 109.469 & 35.806 & 22.48813 & 1.522706 & 190.5641 \\
\hline 17 & 109.404 & 35.666 & 21.78378 & 0.61640 & 193.0289 \\
\hline 18 & 109.424 & 35.551 & 22.06700 & 0.53014 & 229.0574 \\
\hline 19 & 109.457 & 35.435 & 22.58040 & 0.86832 & 257.9343 \\
\hline 20 & 109.472 & 35.300 & 23.21345 & 1.47723 & 269.2503 \\
\hline 21 & 109.515 & 35.166 & 23.27094 & 1.95238 & 284.4721 \\
\hline 22 & 109.544 & 35.021 & 22.47640 & 2.07477 & 298.0574 \\
\hline 23 & 109.577 & 34.882 & 20.53267 & 1.54755 & 299.1291 \\
\hline 24 & 109.688 & 34.771 & 18.89304 & 1.05216 & 268.7144 \\
\hline 25 & 109.823 & 34.685 & 19.47246 & 1.30290 & 239.6672 \\
\hline
\end{tabular}


number of evenly distributed over the interval $(0,1)$. If the random number which obeys normal distribution $X \sim N\left(\mu, \sigma^{2}\right)$ is $X_{i}$, and $r_{i}$ is the random number representing standard normal distribution, the equation is shown in Eq. (10).

$$
\frac{x_{i}-\mu}{\sigma} \sim N(0,1)
$$

Thus, Eq. (11) can be obtained.

$$
x_{i}=\mu+\sigma r_{i}
$$

\section{Case study}

\subsection{MCM for analyzing the uncertainty of environmental parameters}

The ambient temperature changes slowly in space and time. According to the central limit theorem, the error between the true value and the measured value obeys the normal distribution. As shown in Fig. 4, the MCM is used to analyze the uncertainty of environmental parameters at the location of Luochuan $\left(109.537^{\circ} \mathrm{E}, 35.946^{\circ} \mathrm{N}\right)$. In order to combine with the actual line, this paper chooses a $750-\mathrm{kV}$ transmission line from Yuheng, Luochuan to Xinyi according to the geographical wiring diagram of Shaanxi power grid. The transmission line length is $386.7 \mathrm{~km}$. In this paper, we select the latitude range of $109.2^{\circ}-110.0^{\circ} \mathrm{E}$ and the latitude range of $34.6^{\circ}-38.1^{\circ} \mathrm{N}$. The range of longitude span is $80 \mathrm{~km}$ and the latitude span is $350 \mathrm{~km}$, as we can see in Fig. 4a. The environmental parameter data is from the China meteorological data network. We can get the area of a total of $9 \times 36=$ 324 measurement points and the corresponding environmental parameters, as shown in Fig. 4b. In the calculation process, the typical variance values of the temperature, wind speed, and wind direction of each known measurement points are 0.3, 0.5, and 1.0 [17], and the random number corresponding to the normal distribution is acquired by the inverse method. The latitude and longitude and environmental parameters of the 25 known points on the transmission line are given in Table 3.

\begin{tabular}{|c|c|c|c|c|c|}
\hline Points & Longitude (E) & Latitude (N) & Weight of temperature & Weight of wind speed & Weight of wind direction \\
\hline 1 & 109.676 & 37.989 & 0.006944 & 0.008530 & -0.001115 \\
\hline 2 & 109.686 & 37.884 & -0.00294 & -0.001504 & 0.0008418 \\
\hline 3 & 109.729 & 37.744 & 0.002148 & -0.001837 & 0.0000016 \\
\hline 4 & 109.758 & 37.609 & 0.002923 & -0.001088 & 0.0003786 \\
\hline 5 & 109.792 & 37.470 & 0.007261 & -0.0004456 & 0.0006067 \\
\hline 6 & 109.835 & 37.340 & -0.00124 & -0.0003863 & 0.0001889 \\
\hline 7 & 109.864 & 37.244 & 0.006372 & 0.01251 & -0.001392 \\
\hline 8 & 109.844 & 37.095 & 0.001841 & 0.04135 & -0.000122 \\
\hline 9 & 109.820 & 36.970 & -0.01450 & -0.04352 & 0.0003414 \\
\hline 10 & 109.801 & 36.845 & 0.03040 & -0.01894 & 0.0009870 \\
\hline 11 & 109.792 & 36.671 & -0.01211 & -0.0005961 & -0.001447 \\
\hline 12 & 109.758 & 36.570 & -0.05701 & 0.003447 & 0.002044 \\
\hline 13 & 109.739 & 36.416 & 0.1986 & 0.0003571 & -0.000862 \\
\hline 14 & 109.695 & 36.243 & -0.4985 & -0.0006602 & -0.001380 \\
\hline 15 & 109.614 & 36.113 & 0.8586 & 0.5111 & 0.5072 \\
\hline 16 & 109.469 & 35.806 & 0.7825 & 0.4983 & 0.4942 \\
\hline 17 & 109.404 & 35.666 & -0.4213 & -0.002698 & -0.000438 \\
\hline 18 & 109.424 & 35.551 & 0.07082 & -0.002142 & -0.002012 \\
\hline 19 & 109.457 & 35.435 & 0.06238 & 0.004119 & 0.002807 \\
\hline 20 & 109.472 & 35.300 & -0.02833 & -0.001016 & -0.000671 \\
\hline 21 & 109.515 & 35.166 & -0.00514 & 0.002383 & 0.001088 \\
\hline 22 & 109.544 & 35.021 & 0.01500 & -0.02644 & -0.000957 \\
\hline 23 & 109.577 & 34.882 & -0.00392 & -0.01687 & -0.001596 \\
\hline 24 & 109.688 & 34.771 & -0.00001 & 0.03288 & -0.000289 \\
\hline 25 & 109.823 & 34.685 & 0.005737 & 0.003186 & 0.0007310 \\
\hline
\end{tabular}

Table 4 The weight of ordinary Kriging method 


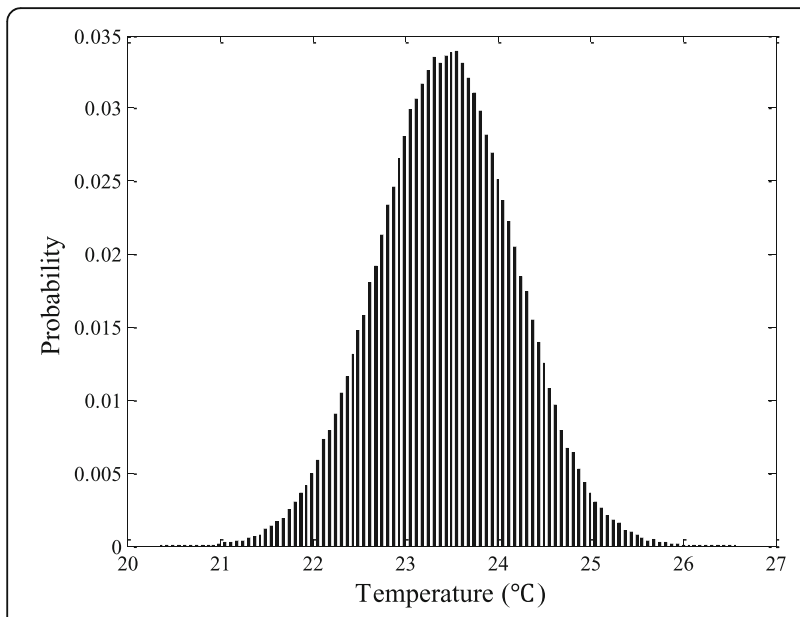

Fig. 5 Ambient temperature distribution histogram

The corresponding weights for the 25 points to get Luochuan parameters by the ordinary Kriging interpolation method are shown in Table 4. The uncertainty of environmental parameters at Luochuan is analyzed by the Monte Carlo method. The histograms of the temperature, wind speed, and wind direction distributions obtained by the MCM are shown in Figs. 5, 6, and 7.

The best estimate value, standard uncertainty, and the shortest confidence interval of 95\% (sampling number $M$ is 200000) are shown in Table 5.

As can be seen from Table 5 , the best estimate value of the MCM is in the shortest inclusion interval with a confidence level of $95 \%$. The standard uncertainty of wind speed is the minimum and the wind direction is the maximum. Among them, the standard uncertainty of wind direction is the largest. And the range of included intervals with the corresponding confidence level of $95 \%$ is also the largest.

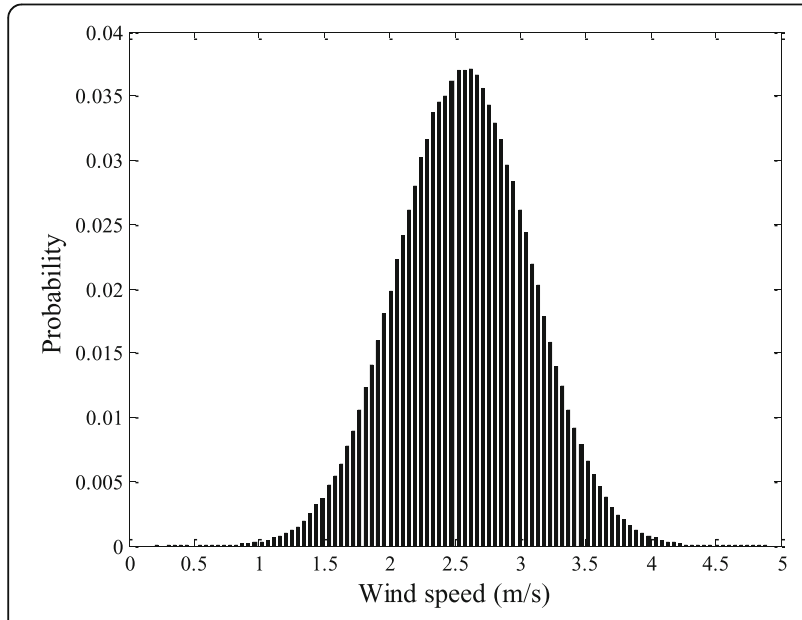

Fig. 6 Wind speed distribution histogram

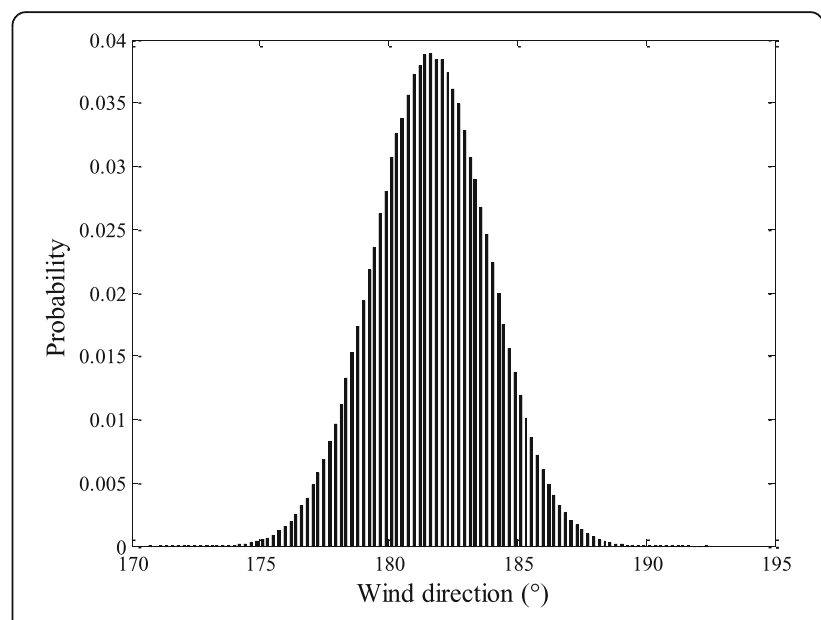

Fig. 7 Wind direction distribution histogram

\subsection{Results and discussions}

From Table 5, we can see that the temperature, wind speed, and wind direction of Luochuan at 8 a.m. on September 17, 2016, are subject to the following distribution.

$$
\left\{\begin{array}{l}
T_{\mathrm{a}} \sim \operatorname{Norm}\left(23.4471,0.5488^{2}\right) \\
V_{\mathrm{w}} \sim \operatorname{Norm}\left(2.6358,0.5072^{2}\right) \\
\phi_{\mathrm{w}} \sim \operatorname{Norm}\left(181.6417,2.2416^{2}\right)
\end{array}\right.
$$

Combined with CIGRE standard, we get the distribution diagram of dynamic thermal rating when the line maximum allowed temperature is $70{ }^{\circ} \mathrm{C}$, as shown in Fig. 8.

Table 6 gives the best estimates of the dynamic thermal ratings obtained from the MCM as well as the standard uncertainty and the minimum inclusion interval with a confidence level of $95 \%$. According to the CIGRE standard based on the environmental

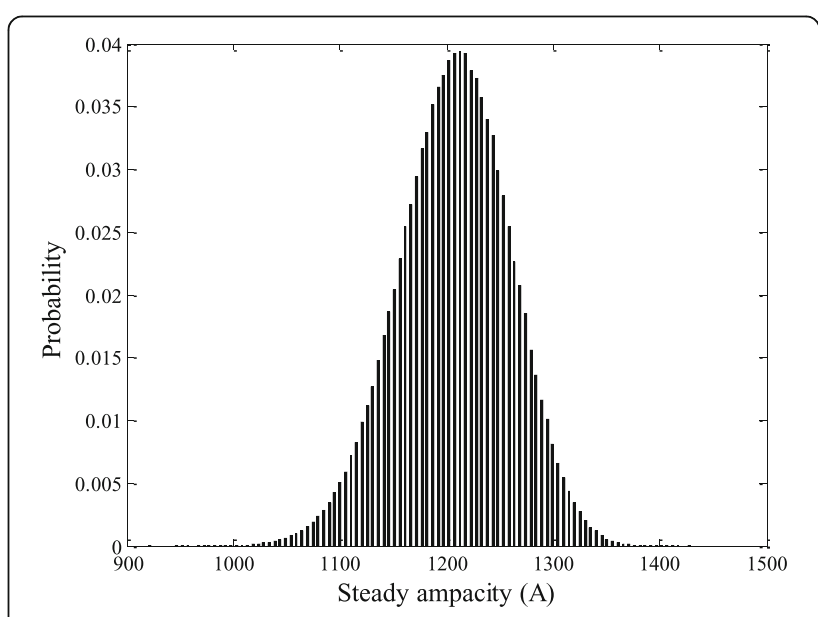

Fig. 8 CIGRE standard DTR distribution 
Table 6 MCM for solving DTR uncertainty

\begin{tabular}{llll}
\hline $\begin{array}{l}\text { Calculation } \\
\text { standard }\end{array}$ & $\begin{array}{l}\text { Best } \\
\text { estimate } \\
\text { value(A) }\end{array}$ & $\begin{array}{l}\text { Standard } \\
\text { uncertainty } \\
\text { (A) }\end{array}$ & $\begin{array}{l}\text { The shortest inclusion } \\
\text { interval with the } \\
\text { confidence level of } \\
95 \%(A)\end{array}$ \\
\hline CIGRE & 1178.5 & 44.7 & {$[1087.3,1262.7]$} \\
\hline
\end{tabular}

parameters, the best estimation values of the dynamic thermal values obtained by the MCM are 1178.5 A, which is in the minimum inclusion interval with a confidence interval of $95 \%$. When the line maximum temperature is $70{ }^{\circ} \mathrm{C}$, static thermal rating of LGJ-400/50 transmission line is 592 A. According to CIGRE standard, the dynamic value of the line can be increased by $83.7-113.3 \%$ with the $95 \%$ confidence level. It can be seen that the dynamic thermal rating can greatly improve the transmission capacity.

\section{Conclusions}

In order to verify the reliability of the DTR of transmission lines, the DTR model based on CIGRE standard is given. The DTR uncertainty is evaluated by MCM method. The application scope and concrete process of the MCM are studied. According to the measurement data of environmental parameters, the estimation uncertainty of ambient temperature, wind speed, and wind direction at 8 a.m. on September 17, 2016, in Luochuan is given. Through Monte Carlo analysis and simulation, the optimal estimation of DTR, the standard uncertainty, and the inclusion interval under the given confidence level are gained. The uncertainty of DTR can be effectively analyzed by MCM method, and the calculation of the line transfer capability is more accurate. Comparing with the static thermal rating obtained by conservative environmental parameters, we find that the DTR technique can increase the line transmission capacity on the basis of the existing transmission lines and improve the efficiency of transmission lines. Future work should study the benefits of dynamic thermal rating.

Table 5 MCM to analyze the output of environmental parameter uncertainty

\begin{tabular}{|c|c|c|c|}
\hline $\begin{array}{l}\text { Environmental } \\
\text { parameter }\end{array}$ & $\begin{array}{l}\text { Best } \\
\text { estimate } \\
\text { value }\end{array}$ & $\begin{array}{l}\text { Standard } \\
\text { uncertainty }\end{array}$ & $\begin{array}{l}\text { The shortest confidence } \\
\text { interval with the confidence } \\
\text { level of } 95 \%\end{array}$ \\
\hline Temperature $\left({ }^{\circ} \mathrm{C}\right)$ & 23.4471 & 0.5488 & {$[21.99,24.90]$} \\
\hline Wind speed (m/s) & 2.6358 & 0.5072 & {$[1.64,3.63]$} \\
\hline Wind direction $\left(^{\circ}\right)$ & 181.6417 & 2.2416 & {$[117.24,186.03]$} \\
\hline
\end{tabular}

\section{Abbreviations}

CIGRE: International Council on Large Electric Systems; DTR: Dynamic thermal rating; GUM: Uncertainty in measurement; MCM: Monte Carlo method

\section{Acknowledgements}

The research presented in this paper was supported by the science and technology development plan of Weihai.

\section{Authors' contributions}

YW is the main writer of this paper. She proposed the main idea. WT introduced the MCM algorithm. RW completed the simulation and analyzed the results. ZY gave some important suggestions for the DTR calculation. All authors read and approved the final manuscript.

\section{Authors' information}

Yanling Wang obtained Bachelor in Architectural Engineering and Master of Electric Power System and Automation from Northeast Electric Power University. She received her Ph.D. degree from Shandong University and now teaches in School of Mechanical Electrical an Information Engineering at Shandong University. Her current research areas are smart grid, power grid transmission capacity, and power system operation and control. Weihua Tao graduated from mechanical electronic engineering of Nanjing University of Science and Technology in 1999. Now he works as engineer in Beaulieu Yarns Weihai Company Limited, Weihai, China. His main field of research is new energy generation (including grid inverter) and large-power electronic circuit.

Zhijie Yan was born in Jiangsu province, China, in 1993. He received his B.E. degree in electrical engineering and automation from Yancheng Teachers University, China, in 2015. He is currently pursuing his M.E. degree in electronics and communication engineering at Shandong University, China. His current research interests include power system operation and control.

Ran Wei received his M.S. degree from Shandong University and now works in Shandong Inspur Software Company Limited. His current research interests include smart grid, power grid transmission capacity, and new energy power generation.

\section{Competing interests}

The authors declare that they have no competing interests.

\section{Publisher's Note}

Springer Nature remains neutral with regard to jurisdictional claims in published maps and institutional affiliations.

\section{Author details}

${ }^{1}$ School of Mechanical, Electrical and Information Engineering, Shandong University, Weihai, China. ${ }^{2}$ Beaulieu Yarns (Weihai) Company Limited, Weihai, China. ${ }^{3}$ Shandong Inspur Software Company Limited, Jinan, China.

Received: 8 March 2018 Accepted: 18 June 2018

Published online: 04 July 2018

\section{References}

1. H Zhang, XS Han, YL Wang, Analysis on current carrying capacity of overhead lines being operated. Power Syst. Technol. 32(14), 31-35 (2008)

2. A Alberto, P Castro, R Martinez etal. Comparison between IEEE and CIGRE thermal behaviour standards and measured temperature on a 132-kV overhead power line. Energies,v 8, n 12, p 13660-71 (2015)

3. G Kosec, M Maksić, V Djurica, Dynamic thermal rating of power lines-model and measurements in rainy conditions. Int. J. Electr. Power Energy Syst. 91, 222-229 (2017)

4. G Coletta, A Vaccaro, D Villacci, A review of the enabling methodologies for PMUs-based dynamic thermal rating of power transmission lines. Electr. Power Syst. Res. 152, 257-270 (2017)

5. YN Feng, SY Li, ZG Han, The verification of GUM gage block measurement uncertainty evaluation. Metrol. Meas. Technol. 33(S2), 62-64 (2013)

6. G Liu, Y Li, S Liu, et al., in Power Engineering Conference. Real-time solar radiation intensity modeling for dynamic rating of overhead transmission lines (IEEE, 2016). St Lucia, Campus, Brisbane, QLD, Australia, Conference date: September 25, 2016 - September 28, pp. 1-6 (2016)

7. TQ Yang, Validation of MCM for GUM uncertainty framework based on MATLAB. Qual. Tech. Superv. Res. 36(6), 32-35 (2014) 
8. J Teh, I Cotton, Reliability impact of dynamic thermal rating system in wind power integrated network. IEEE Trans. Reliab. 65(2), 1081-1089 (2015)

9. A Safdarian, MZ Degefa, M Fotuhi-Firuzabad, et al., Benefits of real-time monitoring to distribution systems: dynamic thermal rating. IEEE Trans. Smart Grid. 6(4), 2023-2031 (2015)

10. S Sediva, M Havlikova, in Proceedings of the 14th International Carpathian Control Conference. Comparison of GUM and Monte Carlo method for evaluation measurement uncertainty of indirect measurements, vol 6 (2013), pp. 325-329

11. CR Black, WA Chisholm, Key considerations for the selection of dynamic thermal line rating systems. IEEE Trans. Power Delivery. 30(5), 2154-2162 (2015)

12. S Karimi, AM Knight, P Musilek, et al., in International Conference on Environment and Electrical Engineering. A probabilistic estimation for dynamic thermal rating of transmission lines. Florence, Conference date: June 7, 2016 - June 10, pp. 1-6 (2016)

13. MA Azpurua, C Tremola, E Paez, Comparison of the GUM and Monte Carlo methods for the uncertainty in electromagnetic compatibility testing. Prog Electromagn Res. B. 34(34), 125-144 (2011)

14. J Yang, MATLAB implementation of Monte Carlo method for evaluating random variables in measurement uncertainty. Metrol. Meas. Technol. 32(14), 51-54 (2012)

15. Y Cao, HY Chen, J Han, Validation of MCM method for uncertainty evaluation of GUM measurement. J. Astronaut. Metrol. Meas. 23(2), 75-78 (2012)

16. HY Chen, Y Cao, J Han, Evaluation of measurement uncertainty based on Monte Carlo method. J. Electron. Meas. Instrum. 25(4), 301-308 (2011)

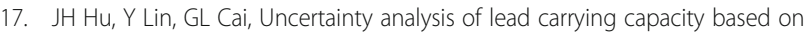
adaptive Monte Carlo method. East China Electr. Power 41(11), 2241-2245 (2013)

\section{Submit your manuscript to a SpringerOpen ${ }^{\circ}$ journal and benefit from:}

- Convenient online submission

- Rigorous peer review

- Open access: articles freely available online

- High visibility within the field

- Retaining the copyright to your article

Submit your next manuscript at $\gg$ springeropen.com 